\title{
Cardiac Magnetic Resonance Imaging of Left Ventricular Apical Hypoplasia in Two Complex Congenital Clinical Syndromes
}

\author{
Matteo Baroni, MD; Patrizia Pedrotti, MD; Stefano Nava, MD;
}

Cristina Giannattasio, MD; Alberto Roghi, MD

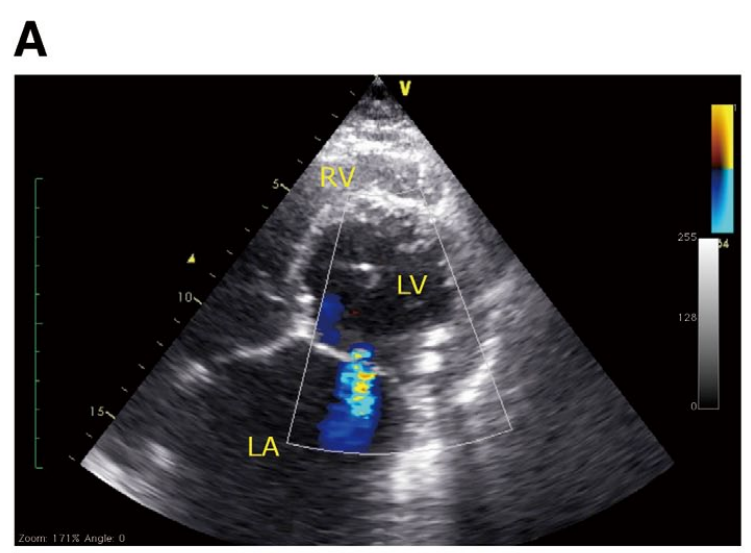

\section{B}

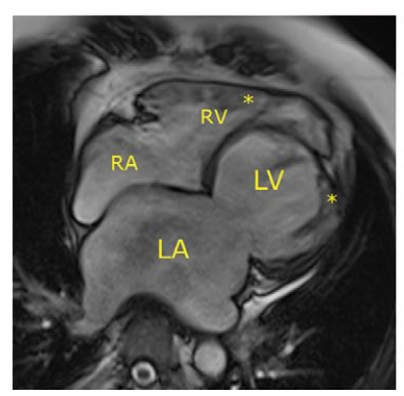

C

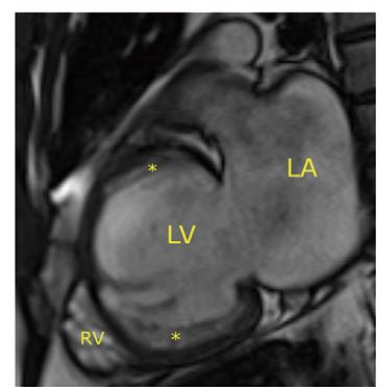

D

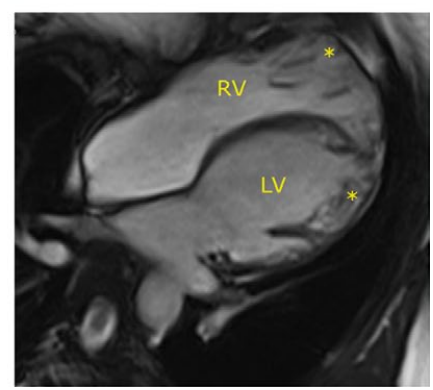

Figure. (A) Color Doppler assessment of mitral regurgitation from transthoracic echocardiography 4-chamber view in patient 1. (B) Magnetic resonance imaging (MRI) of patient 1. Four-chamber image from cine acquisitions, showing typical findings of left ventricular apical hypoplasia (LVAH). (C) Two-chamber image from cine acquisitions. The peculiar aspect of the right ventricle (RV) wrapping around the left ventricle (LV) can be seen. (D) MRI of patient 2. Four-chamber image from cine acquisitions. Deep trabeculae in both ventricles can be observed, in addition to LVAH. LA, left atrium; RA, right atrium. *, areas of non-compaction.

A

45-year-old male patient was referred to the Niguarda Ca' Granda Hospital, Milan, due to worsening dyspnea. He had no familiar history of premature cardiac disease. He had arthrogryposis at birth; congenital amyoplasia (a systemic muscular disease characterized by replacement of skeletal muscle by fatty tissue) was suspected, but no further examination was carried out to confirm the diagnosis. Despite bilateral ankle and hip joint limited mobility, the patient had

Received December 19, 2013; revised manuscript received February 18, 2014; accepted February 20, 2014; released online March 17, 2014 Time for primary review: 33 days

Cardiac MR Laboratory, Cardiology IV, Cardiovascular “A.De Gasperis” Department, Niguarda Ca” Granda Hospital, and Milano-Bicocca University, Milan, Italy

Mailing address: Baroni Matteo, MD, Niguarda Ca' Granda Hospital, Piazza dell’’Ospedale Maggiore 3, Milan, Italy. E-mail: bimatteo@ gmail.com

ISSN-1346-9843 doi:10.1253/circj.CJ-13-1555

All rights are reserved to the Japanese Circulation Society. For permissions, please e-mail: cj@j-circ.or.jp 


\begin{tabular}{|c|c|c|}
\hline First author, year & Age (years)/sex & Concomitant conditions \\
\hline Fernandez-Valls, $2004^{1}$ & $\begin{array}{l}22 / \mathrm{F} \\
46 / \mathrm{F} \\
26 / \mathrm{M}\end{array}$ & None \\
\hline Marin, $2007^{2}$ & 3 months/M & None \\
\hline Flett, $2008^{7}$ & $37 / F$ & None \\
\hline Irving, $2009^{8}$ & $19 / M$ & None \\
\hline Vanhecke, $2011^{3}$ & $53 / F$ & None \\
\hline Haffajee, $2011^{4}$ & $50 / \mathrm{M}$ & Patent ductus arteriosus \\
\hline Chaowu, $2011^{5}$ & $22 / F$ & Complete $\mathrm{AV}$ valves transposition \\
\hline Motwani, $2011^{10}$ & $63 / M$ & None \\
\hline Starmer, $2012^{9}$ & $62 / M$ & None \\
\hline Tumabiene, $2012^{11}$ & $21 / \mathrm{F}$ & None \\
\hline Ong, $2012^{12}$ & $11 / F$ & None \\
\hline Moon, $2013^{6}$ & 33/M & Infundibular pulmonary and aortic stenosis \\
\hline
\end{tabular}

$A V$, atrioventricular; LVAH, left ventricular apical hypoplasia.

a substantially normal life. Due to worsening dyspnea, resting electrocardiogram (ECG) was performed, showing complete left bundle branch block and non-datable atrial fibrillation with normal ventricular rate. Physical examination indicated systolic mesocardial murmur. Rest echocardiography showed spherical left ventricle (LV) with severe depression of systolic function (ejection fraction $[\mathrm{EF}], 30 \%$ ) and mild mitral regurgitation (Figure A); right ventricular function was normal (Tricuspid annular plane systolic excursion, $19 \mathrm{~mm}$ ); estimated pulmonary artery systolic pressure was elevated $(69 \mathrm{mmHg})$. Due to these findings the patient was referred for cardiac magnetic resonance (CMR).

Cardiac magnetic resonance was performed on a 1.5-T scanner (Siemens Avanto, Erlangen, Germany) using a 4-element phased-array receiver coil. All images were acquired with retrospective ECG gating and during repeated single breath-holds. The images acquired showed a spherical LV without a clearly identifiable apex. Wall thickness was diffusely reduced, the interventricular septum was $9 \mathrm{~mm}$ thick, bulging towards the right ventricle. Papillary muscles were abnormal, originating from the truncated apex, and the right ventricle was stretched and extended towards the missing apex (Figures B,C). Right ventricle and the most distal portion of the LV showed areas of noncompaction (trabeculae/wall ratio >2.5). The LV also had severe systolic dysfunction (EF, 30\%) and right ventricular function was mildly reduced (EF, $40 \%)$. Bilateral atriomegalia was also detected and abundant epicardial fat was present above truncated apex. Post-contrast imaging showed a small intramyocardial area of late enhancement at the posterior insertion of the right ventricular free wall in the interventricular septum. Optimal medical therapy for heart failure was initiated, including enalapril, bisoprolol and spironolactone. The patient experienced improvement of symptoms after the onset of therapy although LV function remained severely depressed. The patient refused implantable cardioverter defibrillator and was clinically stable at 2-year follow-up.

A 12-year-old girl was referred for CMR for the diagnostic suspicion of LV non-compaction. She had no family history of congenital heart disease but at the age of 3 months she had undergone operation for ostium primum atrial septal defect causing significant left to right shunt. Subsequently the girl was completely asymptomatic but follow-up echocardiography showed a spherical LV with prominent trabeculae, so CMR was suggested. The examination followed the same study protocol de- scribed in the previous section.

Cardiac magnetic resonance confirmed the truncated aspect of the LV with a thin interventricular septum $(5 \mathrm{~mm})$ bulging towards the right ventricle. Papillary muscles were originating from the flattened apex, which presented very prominent trabecule, compatible with the diagnosis of non-compaction. The right ventricle was stretched, covering part of the missing apex. Diagnostic criteria for non-compaction were fulfilled also for the right ventricle (Figure D). Systolic function was mildly reduced for both ventricles (EF 50\% and 53\% for left and right ventricle, respectively). No epicardial fat tissue was observed near the apex, but small areas of late enhancement were observed in the trabeculae of both ventricles.

The patient was treated with a low dose of $\beta$-blocker (metoprolol $50 \mathrm{mg} /$ day) for anti-arrhythmic prophylaxis and kept asymptomatic during a 4-year follow-up period. The patient's general aspect was mildly dysmorphic, so genetic testing was suggested in the suspect of a syndromic complex, but it was refused by the patient's parents.

Since the first description made in 2004 by Fernandez-Valls et al, ${ }^{1} \mathrm{LV}$ apical hypoplasia (LVAH) has been described in only a few case reports and small series, involving 14 patients globally (Table). ${ }^{1-12}$ Its peculiar features include the following: (1) truncated and spherical aspect of the LV; (2) rightward bulging of the interventricular septum; (3) anomalous origin of papillary muscles from flattened apex; (4) deposition of fatty material contiguous with epicardial fat in the region of the missing apex; (5) elongation of the right ventricle wrapping around the deficient LV apex; and (6) various degrees of systolic dysfunction.

Being found at all ages, including in newborns, ${ }^{2}$ a congenital genesis has been proposed. Many hypotheses have been formulated to explain in utero formation of this pathology but abnormal ventricular septation can be considered the most accepted. ${ }^{1}$ Conversely, another interesting theory suggests that the LV could be divided into 3 distinct anatomic components, namely the inlet; the apical trabecular portion; and the outlet. In this context, isolated LVAH could be considered the expression of congenital malformation of the trabecular portion alone, while the relatively most common hypoplastic left heart syndrome involves all 3 entities. ${ }^{3}$

Moreover, although LVAH has been reported alone in the majority of cases, concomitant congenital conditions were diagnosed in 3 cases, including patent ductus arteriosus, ${ }^{4}$ complete transposition of atrioventricular valves ${ }^{5}$ and combined aortic 
and pulmonary stenosis. ${ }^{6}$

In the present case, 2 complex presentations were found. As best as we could find, none of these conditions have been described before. In the first patient a primary muscular disease was present, albeit not completely clear. Many myopathies are known for various grades of cardiac involvement; and primary amyoplasia, thus still considered idiopathic, has the peculiar histological finding of the replacement of skeletal muscle by fatty tissue. ${ }^{13}$ The same finding is seen in LVAH, where fatty infiltration usually replaces the missing part of the apex. More interestingly, atrial septal defect and biventricular non-compaction were found in the second patient. It is difficult to explain these findings according to the theory of agenesis of the LV portion. Conversely, "classical" LV non-compaction and LVAH share some peculiarities such as various degrees of fatty infiltration into myocardial tissue. ${ }^{14}$ Probably, a common pathological pathway could explain both of the present cardiomyopathy presentations. Although overall prognosis seems to be good with optimal medical therapy for heart failure, current experience is too limited to suggest a standardized therapeutic approach.

Widespread use of CMR has facilitated description of challenging and rare conditions that could be difficult to diagnose properly on ultrasonography, even if recent progress of this technology has narrowed this performance gap over time..$^{15}$ LVAH is one of these challenging pathologies, and its unique features strongly suggest the hypothesis of a new clinical entity. The present findings suggest that LVAH could be ascribed to a form of primary cardiomyopathy, but further observations are needed to better clarify the origin of this disease.

\section{References}

1. Fernandez-Valls M, Srichai MB, Stillman AE, White RD. Isolated left ventricular apical hypoplasia: A new congenital anomaly described with cardiac tomography. Heart 2004; 90: 552-555.

2. Marin C, Sanchez ML, Maroto E, Ossaba S, Ruiz Y, Zabala JI. MR imaging of isolated left ventricular apical hypoplasia. Pediatr Radiol 2007; 37: 703-705.

3. Vanhecke TE, Decker J, Leonowicz N, Chinnaiyan KM. Isolated left ventricular apical hypoplasia. Congenit Heart Dis 2011; 6: 646-649.

4. Haffajee JA, Finley JJ, Brooks EL, Kuvin JT, Patel AR. Echocardiographic characterization of left ventricular apical hypoplasia accompanied by a patent ductus arteriosus. Eur J Echocardiogr 2011; 12: E17, doi:10.1093/ejechocard/jeq170.

5. Chaowu Y, Xin S, Shihua Z, Jianrong L, Hao W. Complete transposition of the atrioventricular valves associated with left ventricular apical hypoplasia. Circulation 2011; 124: e538-e539, doi:10.1161/ CIRCULATIONAHA.111.043620.

6. Moon JI, Jeong YJ, Lee G, Choi JH, Lee JW. Isolated left ventricular apical hypoplasia with infundibular pulmonary and aortic stenosis: A rare combination. Korean J Radiol 2013; 14: 874-877.

7. Flett AS, Elliott PM, Moon JC. Images in cardiovascular medicine: Cardiovascular magnetic resonance of isolated left ventricular apical hypoplasia. Circulation 2008; 117: e504-e505, doi:10.1161/ CIRCULATIONAHA.107.746503.

8. Irving CA, Chaudhari MP. Fatal presentation of congenital isolated left ventricular apical hypoplasia. Eur J Cardiothorac Surg 2009; 35: $368-369$.

9. Starmer G, Younger JF, Stewart P. Multimodality imaging of isolated left ventricular apical hypoplasia. Eur Heart J 2012; 33: 675.

10. Motwani M, Witte KK, Plein S, Greenwood JP. Isolated left ventricular apical hypoplasia evaluated by cardiovascular magnetic resonance and gadolinium enhancement techniques. J Am Coll Cardiol 2011; 58: 2355 .

11. Tumabiene KD, Magpali AE Jr, Chiong L, Jara RD, Abarquez RF Jr, Abelardo NS. A plump and fatty heart: Isolated left ventricular apical hypoplasia. Echocardiography 2012; 29: E193-E196, doi:10.1111/ j.1540-8175.2012.01741.x.

12. Ong CC, Hia CP, Lim TC, Teo LL. Isolated left-ventricular apical hypoplasia presenting as a left-ventricular mass on echocardiography. Pediatr Cardiol 2012; 33: 1456-1457.

13. Rink BD. Arthrogryposis: A review and approach to prenatal diagnosis. Obstet Gynecol Surv 2011; 66: 369-377.

14. Paterick TE, Tajik AJ. Left ventricular noncompaction: A diagnostically challenging cardiomyopathy. Circ J 2012; 76: 1556-1562.

15. Cua CL, Feltes TF. Echocardiographic evaluation of the single right ventricle in congenital heart disease: Results of new techniques. Circ J 2012; 76: 22-31. 